\title{
Improvement of the Parametric Fire of Eurocode 1 based on Experimental Test Results
}

\author{
JEAN-MARC FRANSSEN \\ Univ. of Liege, 6, Quai Banning, 4000, Liege, Belgium \\ jm.franssen@ulg.ac.be
}

\begin{abstract}
The basic equations, the limitations and the main features of the parametric fire proposed in Annexe B of Eurocode 1 are given. 48 experimental fire tests are used to assess the validity of this model. The model has been applied to these tests and the comparison has been made, for each test, on the maximum temperature in the air and on the maximum temperature calculated in 2 hypothetical steel sections, one thermally protected and one unprotected. The agreement is very poor for the air and for the unprotected steel temperature, and somewhat better for the protected steel temperature. Some proposals are made which allow to improve the agreement, while keeping the same expressions for the parametric fire. These modifications concern the equivalent thermal properties of multi material walls, and the introduction of a minimum duration of the fire and a of a ventilation effect in case of fuel-bed controlled fires.
\end{abstract}

KEYWORDS: fire test, parametric fire, comparison, validation

\section{INTRODUCTION}

Nominal fire curves, such as those presented in ISO 834 [1] or ASTM E119 [2], are time temperature relationships which are very useful when used in a grading system, either by experimental testing or by calculation, but they may be a very poor representation of a real fire which could develop in a particular building. They are thus of very little help to assess the behaviour of, say, a critical structural element in a particular building.

Parametric fires provide a simple mean to take into account the most important physical phenomenon which may influence the development of a fire in a particular building. Like nominal fires, they consist of time temperature relationships, but these relationships contain some parameters deemed to represent particular aspects of the reality. In almost every 
parametric fire which can be found in the literature, three parameters are taken into account, in a way or another. Namely:

- the fire load present in the compartment,

- the openings present in the walls and/or in the roof and

- the type and nature of the different walls of the compartment.

Parametric fires are based on the hypothesis that the temperature is uniform in the compartment which limits their field of application to post-flashover fires in compartments of moderated dimensions. They nevertheless constitute a significant step forward toward the consideration of the real nature of a particular fire when compared to nominal fires, while still having the simplicity of some analytical expressions, i.e. no sophisticated computer tool is required for their application.

A proposal is made in the informative annex A of Eurocode 1 - Part 2-2 [3] for such a parametric fire. The aim of this paper is to compare the results provided by this proposal with the result of experimental tests and to make proposals for modifications of the model leading to a better correlation between the experimental results and the results of the calculation.

\section{THE PARAMETRIC FIRE OF EUROCODE 1}

The evolution of the gas temperature in the compartment is given by :

$$
\Theta_{g}=1325\left(1-0.324 e^{-0.2 t^{\circ}}-0.204 e^{-1.7 t^{\circ}}-0.472 e^{-19 t^{\circ}}\right)
$$

with

$$
\begin{aligned}
t^{*} & =\Gamma t \\
\Gamma & =\frac{(O / 0.04)^{2}}{(b / 1160)^{2}} \\
O & =A_{v} \sqrt{h} / A_{t} \\
b & =\sqrt{c \rho \lambda}
\end{aligned}
$$

and

$t \quad$ time, in hour,

$A_{v} \quad$ area of vertical openings, in $\mathrm{m}^{2}$,

$h \quad$ height of vertical openings, in $\mathrm{m}$,

$A_{t} \quad$ total area of enclosure (walls, ceiling and floor, including openings), in $\mathrm{m}^{2}$,

$c, \rho$ and $\lambda \quad$ thermal properties of boundary of enclosure ( $\mathrm{b}$ in $\mathrm{J} / \mathrm{m}^{2} \mathrm{~s}^{1 / 2} \mathrm{~K}$ ).

Equation 1 describes the evolution of the temperature during the heating phase, the duration of which is determined by the fire load according to equation 6 .

$t_{d}^{*}=0.13 \times 10^{-3} q_{t, d} \Gamma / O$

with $q_{t, d}$ design value of the fire load density related to $A_{t}$, in $\mathrm{MJ} / \mathrm{m}^{2}$. 
The temperature-time curve during the cooling phase is given by:

$$
\begin{array}{lll}
\Theta_{g}=\Theta_{\max }-625\left(t-t_{d}^{*}\right) & \text { for } & t_{d}^{*} \leq 0.5 \\
\Theta_{g}=\Theta_{\max }-250\left(3-t_{d}^{*}\right)\left(t-t_{d}^{*}\right) & \text { for } & 0.5 \leq t_{d}^{*} \leq 2.0 \\
\Theta_{g}=\Theta_{\max }-250\left(t-t_{d}^{*}\right) & \text { for } & 2.0 \leq t_{d}^{*}
\end{array}
$$

with $\theta_{\max }$ maximum temperature in the heating phase, for $t^{*}=t_{d}^{*}$.

This parametric fire is valid for compartments up to $100 \mathrm{~m}^{2}$ of floor area, without openings in the roof and for a maximum compartment height of $4 \mathrm{~m}$. $b$ must be in the range 1000 to 2000 , and $O$ must be comprised between 0.02 and 0.20

Figure 1 shows the shape of the temperature-time curve given by equations 1 and 7 . It has been drawn for an opening factor $O$ equal to 0.04 and a wall factor $b$ equal to 1160 . For this case, the heating curve is very similar to the ISO curve. Increasing the opening factor would lead to a shorter but more severe fire. The fire load is $150 \mathrm{MJ} / \mathrm{m}^{2}$, leading to a fire duration of 30 minutes. Modifying the fire load has an influence on the duration of the heating phase.

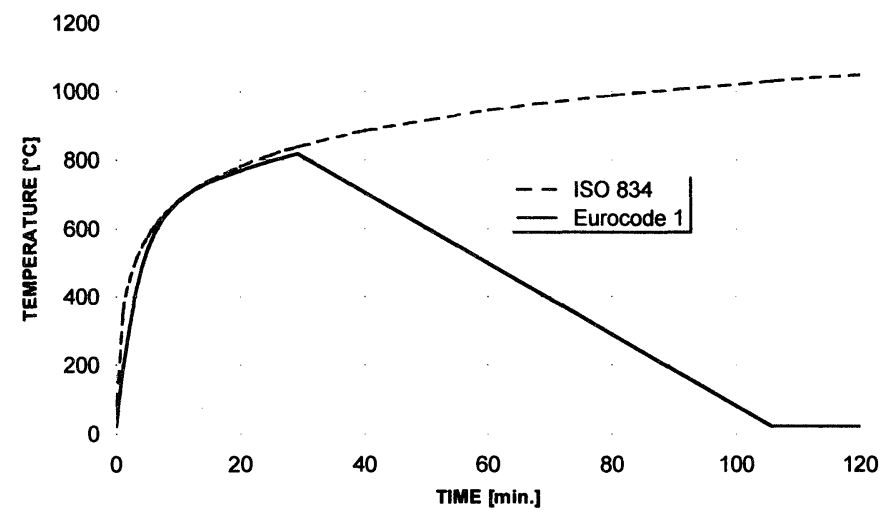

FIGURE 1 : the ISO curve and an example of a parametric fire

\section{COMPARISON OF EC1 WITH EXPERIMENTAL RESULTS}

The results provided by the parametric fire of Eurocode 1 have been compared with gas temperature measurements made during experimental tests [4]. This data base comprises 48 experimental tests made in compartments with a floor area of 12 to $13 \mathrm{~m}^{2}$ and a total volume of 38 to $40 \mathrm{~m}^{3}$. The opening factor $\mathrm{O}$ varies from 0.055 to 0.157 and the fire load qt,d from 38 to $151 \mathrm{MJ} / \mathrm{m}^{2}$. The wall factor $b$ is just at the limit for most of the tests ( 996 for 12 tests and 958 for 24 tests) but it is somewhat smaller for some of them (833 for 3 tests and 751 for 9 tests)

On Figure 2, each test is represented by one point obtained from the maximum temperature in the air calculated by the Eurocode proposal (vertical axis) and measured in the test (horizontal axis). For the tests, the temperature is the average value of several measurement made by 
different thermocouples located in the compartment. The full line on this figure represents the place where all the points should be located if the prediction was perfect. The doted line represents a linear regression of the points.

It appears that, with the test series considered here, there is virtually no correlation between the measured maximum temperature and the maximum temperature predicted by the parametric fire of Eurocode 1. The coefficient of correlation between the experimental results and the calculated results is only 0.23 .

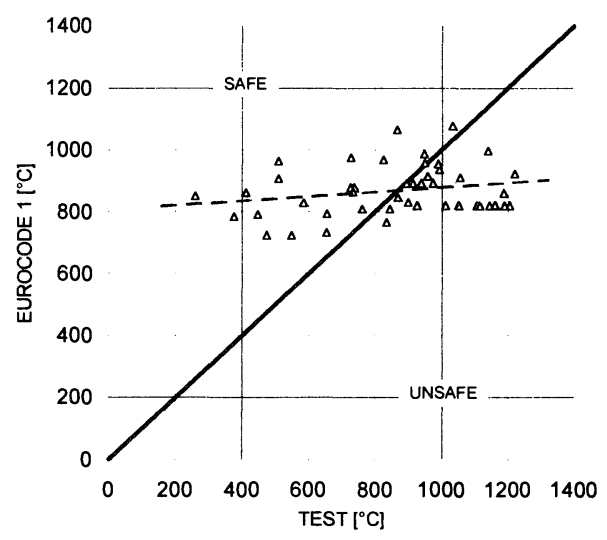

\section{FIGURE 2 : maximum temperature in the air}

The maximum temperature is only a scalar and it hardly represents the whole fire development. It could happen that an otherwise totally bad prediction appears to have the correct maximum temperature and would therefore lead to a point which would be correctly located on Figure 1. In order to address this point, it was decided for each fire to compute the temperature development in an unprotected steel profile having a massivity of $157 \mathrm{~m}-1$, a UB $457 \times 152 \times 74$ for example. The steel temperature has been calculated, first with the air temperature given by the Eurocode parametric fire as an input, then with the air temperature measured in the test as an input. Thermal properties were those of Eurocode 3 [5] and heat transfer conditions were those of Eurocode 1 [3]. The maximum temperature in the steel profile allows some kind of time integration of the evolution of the air temperature. Also, it is the key parameter when it comes to structural stability of the structure. Figure 3 shows the results obtained for the temperature in the unprotected steel profile.

There seems now to be some degree of correlation between the tests and the parametric fire. The higher the temperatures calculated from the experimental measurements, the higher the temperatures calculated from the parametric fire. The correlation is still rather weak, with a coefficient of correlation of 0.69 . 


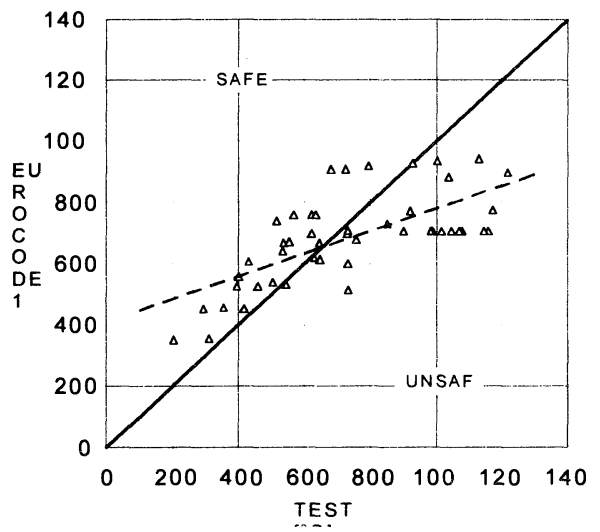

FIGURE 3 : maximum temperature in the unprotected steel profile

In fact, a more detailed examination of the curves showing the evolution of the temperature in the air and the evolution of the temperature in the steel section shows that the curve of the steel temperature generally has a shape very similar to the shape of the air temperature. The steel curve is just lagging some minutes and/or some degrees Celsius behind the gas curve. Therefore, the maximum temperature in the steel section gives the same kind of indication as the maximum temperature in the air concerning the quality of the prediction.

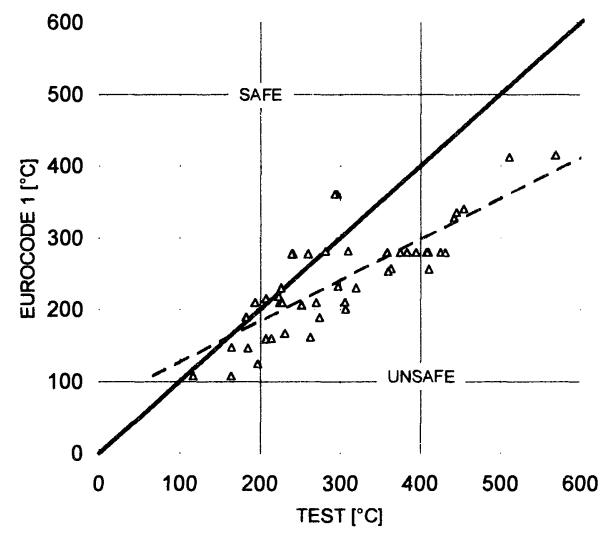

FIGURE 4 : maximum temperature in the protected steel profile

The temperature in a thermally protected profile, on the other hand, is more influenced by the duration of the thermal solicitation, here the fire, than by the maximum temperature reached in the air. Therefore, the evolution of the temperature in the same steel section was computed 
again, with the input coming from the measurement and then from the parametric fire, but this time with a $2 \mathrm{~cm}$ layer of thermal insulation, this material having a thermal conductivity $\lambda$ of 0.20 and a specific heat $\mathrm{c} \rho$ equal to $0.555 \mathrm{MJ} / \mathrm{m}^{3} \mathrm{~K}$, both independent of the steel temperature. The result of this comparison is presented on Figure 4.

The correlation appears now somewhat better, with a coefficient of correlation equal to 0.81 . The Eurocode parametric fire nevertheless leads to temperatures which are almost systematically lower than those produced by the experimental air temperatures, thus leading to an unsafe estimation of the fire resistance time.

\section{MEANS OF IMPROVEMENT}

\section{Properties of the Walls}

In quite a great number of tests from the data base, the walls are made of several layers of different materials. For example, a brick wall insulated by a layer of mineral wool. In case of several layers, Eurocode 1 gives an alternative to equation 5 to calculate the equivalent wall factor, beq. If, in a 2 layers wall, we give the number 1 to the fire exposed side, then the equation of Eurocode 1 is:

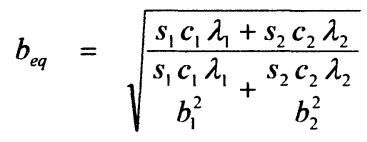

with $s_{l} \quad$ thickness of layer 1 .

There are two major problems with equation 10 .

- The first one is that this equation is symmetric in 1 and 2 . In other words, a wall made of a heavy material insulated from the fire by a lightweight material has the same $b_{e q}$ as a wall with a core made of the lightweight material covered by a layer of the heavy material. It is clear that, in reality, the energy transferred from the hot air to the wall will be mainly influenced by the material which is in direct contact with the air.

- The second problem is that the thickness of each layer plays the same role. Due to this, a wall made of a reasonable thickness of a material 1 covering a very thick material 2 will have an equivalent wall factor almost equal to $b_{2}$ whereas, in reality, this material 2 plays a secondary role.

In order to analyse this effect and make a better proposal, several two layers walls have been analysed with the numerical code SAFIR developed at the University of Liege [6]. Each wall was submitted to the ISO fire and the amount of energy absorbed by the wall was computed. This result was supposed to represent the exact solution. For each case, it was then possible to calculate the material properties of an equivalent wall made of only one material which would absorb the same amount of energy and, therefore, to identify the exact equivalent wall factor beq of the two layers wall. Two different situations emerged from the analyses, depending of the respective values of the wall factor of each material, b1 and b2. Usually, heavy materials are the most heat absorbent. This is why we talk here about heavy and light materials. 
If a heavy material is insulated by a lighter material, it is proposed to calculate the equivalent wall factor as the wall factor of the lighter material, according to equation 11 .

If $\quad b_{1}<b_{2}, \quad b_{e q}=b_{1}$

Figure 5 shows the energy absorbed by the wall after 30 minutes of fire for a normal weight concrete wall $(b 2=2000)$ insulated by a layer of mineral wool $(b 1=200)$, calculated for different thickness of the mineral wool. It shows how the Eurocode 1 formula overestimates the absorbed energy due to the fact that it gives an overwhelming importance to the concrete whereas, in fact, this material is hidden by the mineral wool. The new proposal given in equation 11 systematically underestimates the absorbed energy and is, therefore, on the safe side. It can be seen that, as soon as a reasonable amount of insulating cover is provided, the assumption is also reasonably good.

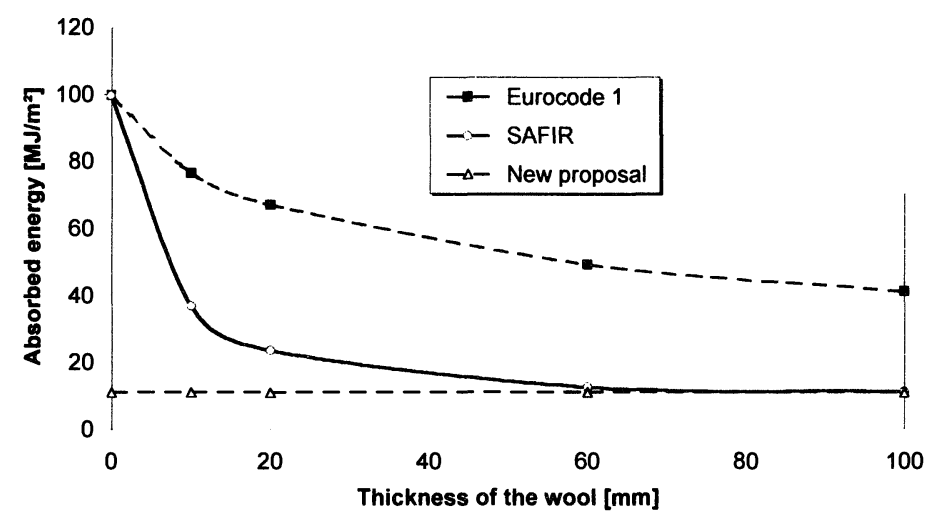

FIGURE 5 : insulating material in contact with the fire

If a light material is covered by a layer of heavier material, like in a sandwich panel for example, it is proposed to use the following procedure to calculate the equivalent wall factor.

If $\quad b_{1}>b_{2}$,

a limit thickness $s_{\text {lim. } I}$ is first calculated for the exposed material according to

$s_{\text {lim, }}=\sqrt{\frac{t \lambda_{1}}{c_{1} \rho_{1}}}$

with $t$ the time of the heating phase of the fire, in seconds.

If

$$
\begin{aligned}
& \text { If } \quad s_{1}>s_{\text {lim }, 1} \text { then } \quad b_{e q}=b_{1} \\
& \text { If } \quad s_{1}<s_{\text {lim }, 1} \text { then } \quad b_{e q}=\frac{s_{1}}{s_{\text {lim }, 1}} b_{1}+\left(1-\frac{s_{1}}{s_{\text {lim }, 1}}\right) b_{2}
\end{aligned}
$$

Figure 6 is drawn for a case which is perhaps academic but has been chosen because it is very illustrative. This is a wall made of lightweight concrete covered, on the inside face, by a layer 
of normal weight concrete (N.W.C. on the figure). The figure shows that the Eurocode formula almost totally neglects the contribution of the N.W.C. whereas the exact SAFIR calculation shows that it is by far not negligible. The new proposal allows to calculate a limit thickness of the first layer slim, 1 equal to $60 \mathrm{~mm}$. The influence of the first material increases with its thickness, up to the limit thickness when the influence of the second material is totally disregarded, because it has become negligible. The second material is totally hidden by the first one.

Other comparisons on more practical cases have shown that, for an insulating sandwich panel with steel sheets, the underestimation made by the Eurocode formula is not as severe as the one shown on Figure 6, but the proposed new formula is nevertheless better, leading to results almost equivalent to the exact calculations. For a gypsum board panel with mineral wool inside, The Eurocode 1 formula amounts to almost totally neglect the contribution of the gypsum board, whereas the results provided by the new proposed formula are very close to the exact solution.

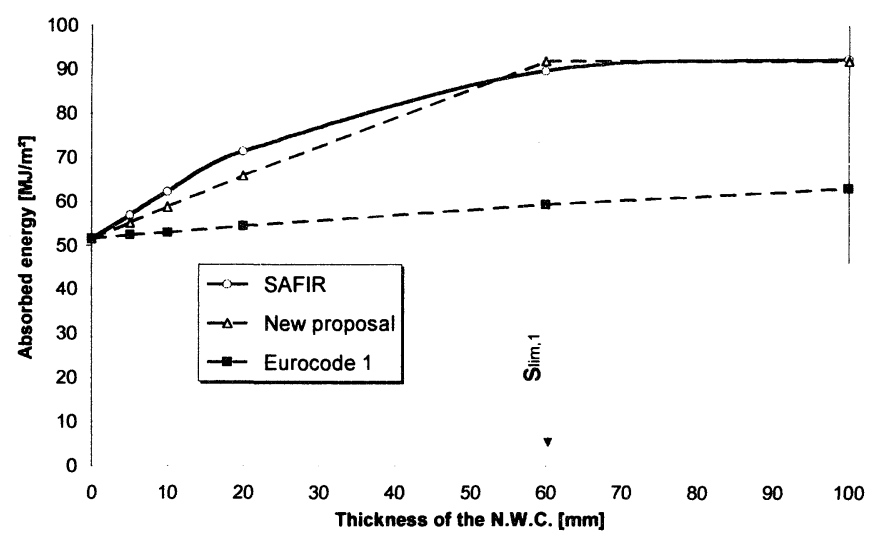

\section{FIGURE 6 : heavy material in contact with the fire}

For this test series, wall factors calculated according to equation 11 instead of equation 10 changed from 958 to 469 ( 24 tests), from 833 to 400 ( 3 tests) and from 958 to 469 ( 24 tests) while they remained unchanged to 996 for 12 tests. The fact that the correlation is improved leads in fact here to a wider field of application of the model concerning the wall factor.

\section{Fuel Controlled Fires}

One of the main hypotheses on which the parametric fire of Eurocode 1 is based is the assumption that the energy release during the fire is directly related to the opening factor. In other words, the fire is ventilation controlled. Indeed, considering that the heat content of the fuel is $16 \mathrm{MJ} / \mathrm{kg}$ and that $70 \%$ of the fuel load has been burnt at the end of the heating phase, Equation 6 can easily be transformed into the following one: 
where $R$ is the burning rate in $\mathrm{kg} / \mathrm{h}$.

Equation 15 is the typical equation often quoted as Kawagoe's equation for the burning rate in an air controlled fire.

When the ratio between the fire load and the opening factor decrease, Equation 6 leads to unrealistic very short fire duration. Based on the fact that any object or fire load needs a certain amount of time to burn, even in the unlimited presence of air, our proposal is to modify equation 6 and replace it by equation 16 .

$t_{d}=\min \left(0.13 \times 10^{-3} q_{t, d} / O ; t_{\text {lim }}\right)$

with tlim chosen as 20 minutes in this paper, similar to the free burning fire duration assumed in Annexe $\mathrm{C}$ of Eurocode 1-2-2 [3].

When applying equation 16 , two different possibilities exist.

Either the duration of the heating phase of the fire calculated from the first term of the equation, $0.13 \times 10^{-3} q_{t, d} / O$, is larger than the chosen limit time $t_{\text {lim. }}$ in which case equations 1 to 4 and equations 7 to 9 are applied as such, without any modification.

Or the duration of the heating phase of the fire calculated from the first term of the equation, $0.13 \times 10^{-3} q_{t, d} / O$, is shorter than the chosen limit time $t_{\text {lim }}$. In this case, equations 1 to 4 are applied with a modified opening factor, $O_{\text {lim }}$, calculated as the one leading to the chosen limit time from the following equation:

$O_{\text {lim }}=0.13 \times 10^{-3} q_{t, d} / t_{\text {lim }}$

Equation 2 and 3 are modified in the following way:

$t_{\lim }^{*}=\Gamma_{\lim } t$

$\Gamma_{\lim }=\frac{\left(O_{\lim } 0.04\right)^{2}}{(b 1160)^{2}}$

and $t_{\text {lim }}^{*}$ is used in equation 1 instead of $t^{*}$.

Applying equation 1 up to the limit time $t_{\text {lim }}$ allows to calculate the maximum temperature of the heating phase. Equation 7 to 9 which give the temperature evolution during the cooling phase are not modified. $t^{*}$ is used in these equations, and not $t_{\text {lim }}^{*}$. This is because the venting and cooling of the compartment when the fire has stopped has nothing to do with the fact that the fire was air controlled or fuel controlled during the heating phase.

\section{Ventilation during the Heating Phase}

The limit opening factor $O_{\text {lim }}$ that has been introduced in the above paragraph allows to slow down the fire in case of large openings, because not all the air entering through the openings is used for combustion. This modification slows down the fire and reduces the temperature level. There is yet another influence of the openings which is present when the fire is fuel controlled; the amount of gas passing through the openings is higher than what the fictitious 
opening factor $O_{\text {lim }}$ tends to indicate. The mass exchange between the compartment and the exterior is higher with large openings than with small openings. A more important part of the energy produced by the fire is therefore evacuated outside by mass transfer, and this also tends to limit the elevation of the temperature in the compartment.

In order to take this effect into account, the following modification is proposed when the duration of the heating phase of the fire calculated from the first term of equation 16 , $0.13 \times 10^{-3} q_{t, d} / O$, is shorter than the chosen limit time tlim .

If $O>0.04$ and $q_{t, d}<75$ and $b<1160$

then $k=1+\left(\frac{O-0.04}{.04}\right)\left(\frac{q_{t, d}-75}{75}\right)\left(\frac{1160-b}{1160}\right)$

and $\Gamma_{\lim }=k \frac{\left(O_{\lim } 0.04\right)^{2}}{(b 1160)^{2}}$

Although this modification is based on a physical phenomena, it has to be recognised that the shape and the coefficients of equation 20 have been derived more from a curve fitting exercise with the results of the data base than from theoretical considerations.

\section{COMPARISON OF THE MODIFIED PARAMETRIC FIRE WITH EXPERIMENTAL RESULTS}

If the parametric fire of Eurocode 1 is modified according to the proposals presented in the preceding section, the comparison can be made between the results of experimental test and the results of the (hopefully) improved prediction. Figure 7 concerns the maximum temperature in the gas and has to be compared to Figure 2 . The coefficient of correlation, which had the value of 0.23 with the Eurocode 1 parametric fire, has now a value of 0.84 .

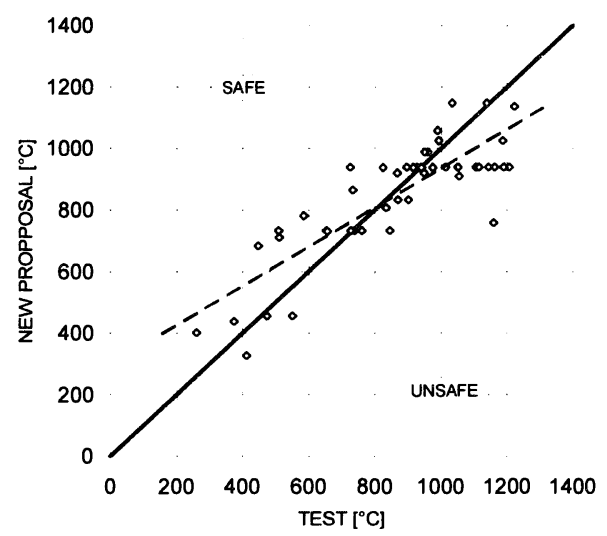

FIGURE 7 : maximum temperature in the air 
Figure 8 has been drawn from the calculated temperatures in the unprotected steel section and has to be compared to Figure 3. The coefficient of correlation has been improved from 0.69 for the original Eurocode proposal to 0.77 for this new proposal.

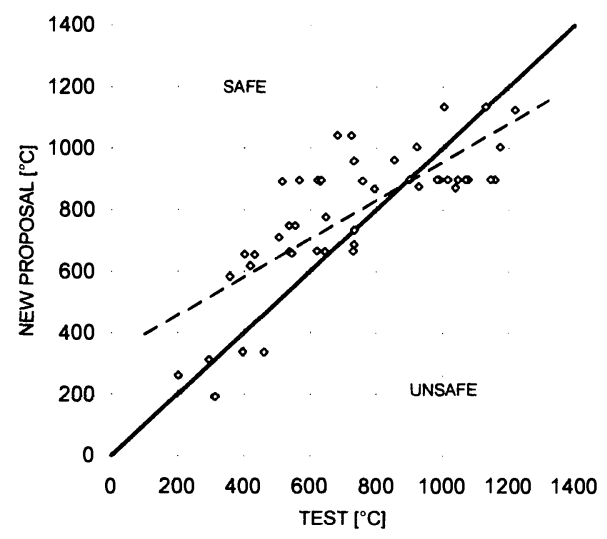

FIGURE 8 : maximum temperature in the unprotected steel profile

The comparison on the temperature of the protected steel profile is presented in Figure 9, to be compared with Figure 4 . The coefficient of correlation is marginally modified, from 0.81 to 0.80 , but, more important, the results are not any more systematically on the unsafe side, see Figure 4, but rather on the safe side.

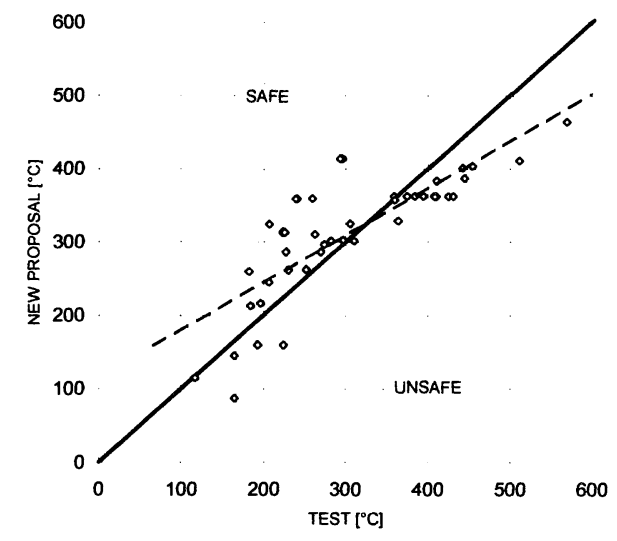

FIGURE 9 : maximum temperature in the protected steel profile 


\section{CONCLUSIONS}

The maximum temperature in the compartment predicted by the parametric fire proposed in Annex B of Eurocode 1 does not correlate at all with the maximum temperature recorded in experimental tests. There is a weak correlation when the maximum temperature in an unprotected steel profile is considered and a better correlation when the temperature in a protected steel profile is considered. In this case, however, the predicted temperature is almost systematically lower than the recorded temperature.

A new proposal has been made to improve the situation. It is based on the same analytical expressions as the original Eurocode proposal but introduces some modifications for the consideration of multi layers walls and for fuel controlled fires. With this new proposal, the correlation between the calculations and the measurements is significantly improved and the results are more on the safe side.

Work is still under way on this subject because more experimental test results which have been collected have still to be introduced in the data base and taken into account. New experimental tests are also currently being made in Europe. An improved new proposal could be made in the near future if it proves to enhance the prediction even more.

\section{ACKNOWLEDGEMENTS}

The work described in this paper was undertaken within the C.E.E. sponsored research "Natural Fire Safety Concept". The author wants to acknowledge participation of other persons in this work, namely J. B. Schleich and L. G. Cajot from ARBED Research, J. F. Cadorin from Univ. of Liege, D. Joyeux from CTICM, S. Pustorino from CREA and G. Aurtenexte from LABEIN.

\section{REFERENCES}

[1] Fire Resistance Tests - Elements of Building Construction. International Standard 834, ISO, 1975.

[2] Standard Methods of the Fire Tests of Building Construction and Materials, Designation E1 19-95a, ASTM, Philadelphia, 436-456, 1995.

[3] Eurocode 1 - Basis of design and actions on structures - Part 2-2: Actions on structures - Actions on structures exposed to fire. ENV 1991-2-2, CEN, Brussels, Fébr. 1995.

[4] J. B. Schleich, L. G. Cajot \& M. Pierre, Natural Fire Safety Concept, Techn. Report $\mathrm{n}^{\circ}$ 7, Semestriel Report, Period from 01.07.97 to 31.12.97, Profil ARBED, 1998.

[5] Eurocode 3: Design of steel structures. Part 1.2: General rules. Structural fire design. Draft ENV 1993-1-2, CEN, Brussels, may 1995

[6] J. M. Franssen, Contributions à la modélisation des incendies dans les bâtiments et de leurs effets sur les structures, Thèse d'agrégation de l'enseignement supérieur, Univ. de Liège, pp. 484, 1997. 